\title{
Téoros
}

Revue de recherche en tourisme

\section{Origines et perspectives historiques de l'accueil}

\section{Pierre Gouirand}

Volume 10, numéro 2, juillet 1991

Accueil et tourisme

URI : https://id.erudit.org/iderudit/1078954ar

DOI : https://doi.org/10.7202/1078954ar

Aller au sommaire du numéro

Éditeur(s)

Université du Québec à Montréal

ISSN

0712-8657 (imprimé)

1923-2705 (numérique)

Découvrir la revue

Citer cet article

Gouirand, P. (1991). Origines et perspectives historiques de l'accueil. Téoros, 10(2), 3-9. https://doi.org/10.7202/1078954ar d'utilisation que vous pouvez consulter en ligne.

https://apropos.erudit.org/fr/usagers/politique-dutilisation/ 


\section{Origines et perspectives historiques de l'accueil}

\section{L'homme est une structure d'accueil}

L'accueil a pris dans la société moderne une place que rien jusqu'ici ne permettait de prévoir.

Il semble que l'on ait brusquement réalisé que pour pallier le manque de sociabilité de la plupart des gens et pour compenser les ravages de l'égoïsmeet de l'individualisme, qui comme le dếnonçait déjà Tocqueville, sont les deux fléaux des sociétés égalitaires, il fallait que la notion d'accueil s'introduise dans la plupart des circonstances de la vie, alors qu'elle était auparavant réservếe au domaine des voyages et des déplacements.

L'accueil qui était une médiation est devenu une chose, une marchandise que l'on vend et qui sert à vendre. Il est à la fois instrument et produit.

Cet accueil a pris une telle importance qu'il modifie le comportement d'achat du client et qu'il laisse des traces bien au-delà du moment de la relation. Telle personne ne reviendra jamais dans tel établissement parce qu"elle y a été mal reçue.

Pourtant, l'homme est biologiquement et cérébralement une structure d'accueil. II ne vit pas en société par goût ou par habitude mais par nécessité vitale. Comme les animaux, il possède des mécanismes d'accueil innés et instinctifs, toutefois, il $n$ 'agit pas comme l'animal en vertu d'automatismes inconscients mais en fonction d'habitudes sociales.

Au cours de l'histoire, ces habitudes ont évolué. Les grandes migrations, le choc des cultures, le progrès technique, enfin tout ce qui a fait changé le monde a produit partout de nouvelles moeurs. En revanche, certains comportements naturels sont restés à peu près semblables et l'accueil semble être de ceux-là.

S'ilapris des formes différentes, il est, dans le fond, resté le même.

\footnotetext{
Monsieur Pierre Gouirand, docteur es lettres, est président de I'Instutut de prospective du tourisme. II est egalement directeur general de I'Hotel Westminster Concorde a Nice.
}

Le processus d'accueil est fait de l'enchaînement de 3 concepts fondamentaux:

- la reconnaissance;

- l'hospitalité:

- le maternage.

Nous allons voir comment ils se retrouvent dans différentes situations d'accueil à travers les âges.

Le philosophe Hegel considère que la reconnaissance est pour l'homme la condition de son existence psychique.

L' homme est conscient de soi, dit-il, de sa réalité, de sa dignité et c'est en cela qu'il differe de l'animal.

Il prend conscience de soi quand il dit «moi» et il est rappelé à lui par le désir, le désir de manger par exemple. C'est ce désir qui le constitue parce que c'est lui qui désire et pas un autre.

Mais ce moi est animal tant que le désir porte sur quelque chose de naturel: de la nourriture par exemple. Pour que le désir devienne humain, il faut qu'il porte sur quelque chose quí dépasse la réalité matérielle; or ce qui dépasse le mieux de cette réalité c'est essentiellement le désir luimềne.

Ainsi, le désir qui đésire un autre désir crée un moi humain différent d'un moi animal et dans l'accueil, ce que l'on désire, c'est être désiré.

Tout homme en situation d'être accueilli souhaite être reconnu comme celui que l'on désire recevoir et veut qu'on le lui montre.

Si l'accueil est biologiquement naturel, en revanche la notion d'hospitalité semble être étrangère à la nature humaine. L'homme modeme, en gếnếral, doit se forcer et faire appel à la morale et au sentiment de solidarité pour être hospitalier.

C'est pourquoi l'hospitalitế est souvent présentée comme bénéfique pour celui qui la pratique.

\section{Des origines divines et religieuses}

Celui qui est hospitalier s'identifie à Dieu qui reçoit tous les hommes de bonne volonté.

On ne connaît pas l'origine des "Lois" de 1 'hospitalité. Elles n'ont jamais fait l'objet d'une rédaction claire, et elles procèdent de la tradition orale. On en trouve trace dans labible, chez Homèreet chez Virgile qui les attribue à Jupiter: «Jupiter... c'est à toi que nous devons les lois de l'hospitalités (l'énểide livre I).

Ce qui est certain, c'est qu'à une ếpoque où le droit international n'existait pas, ou tout au moins où il n'ćtait pas codifié, et où malgré l'inconfort des routeset des moyens de transports les voyages ếtaient tout de même nombreux. Il fallait bien assurer la sécurité des voyageurs etdes individus hors de leur pays d'origine.

En l'absence d'auberges, on devait leur garantir le gîte, le couvert et la sécurité. Un voyageur sans abri était un voyageur mort. Non seulement sa vie étant en danger mais encore il ne pouvait trouver sur sa route aucune provision de bouche. Le rejeter était le vouer à une mort certaine. Il a donc bien fallu trouver un moyen pour contraindre ceux qui se trouvaient sur son chemin, à le recevoir.

Comme aucun moyen de coercition ne pouvaits "exercer, seule la morale ou la religion pouvait obliger les peuplesầture hospitaliers et à recevoir l'étranger de passage et c'est pourquoi le voyageur a été divinisé.

Les lois de l'hospitalité firent leur apparition et plus tard, des divinités spéciales, Zeus Xénios et Athéna Xenia par exemple à Athènes, veillentà leur stricte observance. Leur pratique procure à celui qui les respecte considération et bienfaits moraux car donner l'hospitalité, c'est s'acquitter deses devoirs envers les Dieux. Ces lois, bien que non écrites, sont arrivées plus ou moins exaciement jusqu"à nous.

On devait au voyageur le logis et la nourriture. Le premier jour, on l'invite à la table de la famille, ensuite on lui envoie des provisions. Lors de son départ, on lui fait 
des cadeaux, on échange avec lui des signes de reconnaissance qui pourront se transmettre de génération en génération et qui permettront aux descendants de l'un ou de l'autre de recevoir l'hospitalité dans la maison de celui qui possède le même signe de reconnaissance. Ces signesétaient habituellement des objets en deux parties -

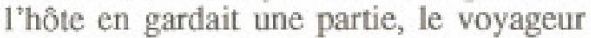

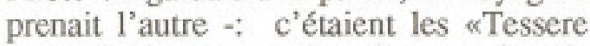
d'hospitalité sous I'empire romain. Ils constituaient de véritables pactes d'hospitalité entre deux familles ou deux groupes.

Si, par malheur, un voyageur hôte d'une maison venait à mourir, on devait également pourvoir à sa sépulture. Ces alois contraignaient en quelquesorte àagir vis-âvis d'un voyageur étranger comme on aurait agit vis-à-vis de quelqu'un de sa propre famille.

Le contenu de l'hospitalité est assez bien expliqué par son origine religieuse.

Fustel de Coulange ${ }^{(1)}$ voit cette origine dans les rites sacrificiels de la religion des premiers temps. Il remarque qu'au tout début de la cité, la seule religion est celle de la famille ou de la phratrie, autrement dit, celle des ancêtres. Il $n^{t} y$ a pas encore de religion publique ou officielle, la religion se transmettait par le sang, c'etait l'appartenance à la famille ou au clan qui faisait de I'adolescent un membre de la asectes et qui lui permettaitde participer au culte. Son admission dans la communauté avait lieu sous forme religieuse. Le jeune était présenté par son père, on immolait une victime et on en faisait cuire la chair sur

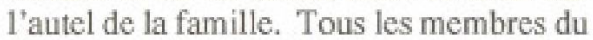
clan familial étaientpresents. S'ilsdoutaient. de la légitimité du jeune homme et refusaient de l'admettre, ils devaient enlever la chair de dessus l'autel, s'ils ne le faisaient pas et qu'après la cuisson, ils partageaient la chair de la victime avec le nouveau venu, c'est que celui-ci était admis dans la phratrie, le clan familial.

Fustel de Coulange note que ce qui expliquait ces pratiques, c'est que les anciens croyaient que toute nourriture préparée sur un autel et partagée entre plusieurs personnes établissait entre elles un lien indissoluble et une union qui ne cessait qu'avec la mort.

C'étaitcette croyance qu'il place à l'origine du contenu de l'hospitalité.

Il pense que l'homme qui, en voyageant, protégé par les lois de l'hospitalité avait réussi à atteindre le foyer d'une famille et en avait partagé la nourriture, ne pouvait plus êture fegardé comme un étranger, ilếtait regardé comme un frère. Celui qui avait partagé le repas sacré était pour toujours en communauté religieuse avec son hôte.

Il souligne également le paradoxe de la situation. La religion domestique n'est pas faite pour l'étranger: par essence, elle le repousse, mais par cela mêne, l'étranger qui y est admis est d'autant plus sacré. Dès qu'il a touche le foyer, il faut de toute nécessité qu'il cesse d'être un étranger. Le principe qui l'écartait de la famille exige qu'il en soit membre et pour toujours. A ce titre, il acquiert alors les mêmes droits que les membres de la famille. Il ćtait protégé par tout le clan, lui nuire était nuire au clan.

Notre mot shôtes vient du latin hospes qui signifie également celui qui reçoit comme celui qui est reçu. Mais hospes est lui-même tiré d'un autre mot latin hostis (qui a donné hostile en français), dont le premier sens est étranger et deuxième sens ennemi.

$\mathrm{L}^{+}$étranger qui est l'ennemi, devient, par la vertu de l'hospitalité, l'hôte, le frère, le membre de la famille que l'on doit nourrir, aimer et protéger.

C'est le grand paradoxe de l'hospitalité: elle fait de l'ennemi un ami, de l'inconnu un frère.

\section{Naissance de I'hospitalité publique}

Avec la formation de la cité est née l'hospitalité publique, extension artificielle de l'hospitalité privée des temps primitifs. Elle a pris sa forme avec l'institution de la Proxénie en Grèce, qui n'est pas sans analogie avec l'institution des aconsuls honoraires» dans nos sociétés.

Le Proxène était le citoyen d'une cité, qui, en vertu d'un contrat passé avec une autre cité, hébergeait, protégeait et défendait les intérêts des citoyens de cette cité (dont il était le Proxène) lorsque ceux-ci avaient affaire chez lui dans sa propre cité natale.

Ainsi, les êtrangers qui auraient dî être des ennemis, étaient acceptés, puisqu'ils vivaient sous la protection et avec la garantie d'un citoyen de la cité.

Il faut noter que seuls ceux qui étaient prodigieusement riches pouvaient être proxènes car l'obligation de fournir" l'hospitalitế pouvait être fort onéreuse. On garde le souvenir d'un sicilien qui dut recevoir et nourrir cinq cents chevaliers, et qui, à leurdepart, leur rem it des manteaux et des tuniques.

Le principe de l'hospitalité publique a survêcu jusqu'à nos jours.
Dans certaines ethnies, comme chez les Kabyles, ou dans certaines parties de la Turquie, les hôtes étaient, il y a encore peu de temps, pris en charge par la communauté

Aujourd 'hui, lorsqu'un pays, une administration ou une municipalité invite une personnalité, il pratique l'hospitalité publique.

Celle-ci s'exerce également dans un autre domaine: celui des hốpitaux, où l'État prend en charge ceux qui en ont besoin.

Nous rejoignons là encore le sens profond du mot: le hospes latin, outre la famille du mot hôte, hôtel, hôtellerie, a donné les mots hôpital (hospital en anglais), hospice et 1 'adjectif hospitalier.

\section{Les concepts d'hospitalité}

Parmi les nombreuses interprétations du concept d'hospitalité, nous en retiendrons particuliêrement deux:

\section{1) Celle de Kant:}

Le philosophe allemand du $18 \mathrm{eme}$ siècle définit l'hospitalité comme le droit d'un étranger à ne pas être traité hostilement lors de son sejour dans un autre pays.

Il fonde même le droit international sur les relations d'hospitalité «le droit cosmopolite doit se restreindre aux conditions de l'hospitalité universellex(2).

Toutefois, il limite le droit ă l'hospitalitế de deux manières:

- on n'est tenu de recevoir quelqu'un qui le demande que s'il est réellement en danger, won peut ne pas le recevoir si cela n'entraîne pas sa ruines;

- on ne doit protection qu'autant que le visiteur n'est pas hostile: s'il ne se tient pas paisiblement à sa place, on est relevé de sa promesse.

Il le limite aussi dans le temps en le qualifiant de droit de visite, alors que l'ctranger qui voudrait s'installer dans une maison pour quelques temps exigerait, dit-il an contrat particulier de Bienfaisances.

Mais surtout, et sous réserve de ce qui précède, il considère l'hospitalité comme un droit naturel. Le voyageur est revêtu d'un droit qui lui permet d'exiger qu'on lui donne $1^{t}$ hospitalite; celle-ci ne résulterait donc plus uniquement de la bonne volonté de celui qui recoit. 
Cette conception renforce considérablement la position de celui qui arrive. Il n'est plus un solliciteur. Il secontented'exiger son dũ et de faire valoir son droit.

$\mathrm{C}^{\gamma}$ est une attitude que $l^{z}$ on rencontre très souvent dans la vie moderne. Instinctivement, mème quand ils n'ont jamais lu Kant, la plupart des gens ont le sentiment «qu'ils ont le droit d'être bien reçus».

\section{2) Celles des encyclopédistes français ${ }^{(3)}$ :}

Ils définissentl'hospitalité comme la svertu d'une grande âme qui tient à tout l'univers par les liens de l'humanités et, disent-ils, plus loin, cette vertu est: «une libéralitét exercée envers les étrangers.....

Ils y voient donc le moyen pour l'homme de dépasser son champ d'action habituel et d'être en relation avec le monde entier. Par l'hospitalité, qui est un facteur de paix mondiale, l'homme s'universalise.

Mais aussi, tout comme Kant, ils restreignent le droit à l'hospitalité, alors que I'antiquité, en faisant protéger le voyageur par la divinité n'y mettait aucune bome. «Pour être tenu par la loi naturelle aux. services de l'hospitalité pris dans toute leur étendues dit-on dans l'article ehospitalité de l'encyclopédies, il faut:

- que celui qui les demande soit hors de sa patrie pour quelque raison valable ou du moins innocente;

- qu'il y ait lieu de le présumer honnête homme, ou du moins qu'il n"ait aucun dessein de porter préjudice à celui qui l'héberge;

- enfin, qu'il ne trouve lui-même pas ailleurs, ou qu'on ne trouve pas à le loger ailleurs pour de l'argent.

Ces quelques lignes appellent un commentaire: on doit tout d'abord noter l'emploi de l'expression «Loi naturelle», différente du droit naturel de Kant, elle implique que l'homme avaitl'obligation d'être hospitalier et de secourir son prochain en voyage.

Mais cette loi ne s"applique pas dans tous les cas, et les krègles* d'application en sont. plus restrictives que celles de Kant.

Il faut être khors de sa patrie»: sans doute faut-il l'entendre au sens large, c'est-à-dire hors de son lieu de résidence habituel.

Il faut encore que la raison du voyage soit «valable ou du moins innocentes.

Ces termes sont ambigus. Qu'est-ce qu'une raison valable?
Il est vraisemblable que cette règle a été tirée de l'usage de l'époque. Celui qui voyageait sans motivation avouéc et sans but précis était un peu suspect. Partir pour le plaisir de partir n'était pas encore passé dans les moeurs.

On retrouve cette idée sécuritaire dans la deuxième règle. Le voyageur dit être "présumé honnête homme» et n'avoir saucun dessein de porter préjudice à celui qui l'héberges. C'est évidemment une règle de bon sens. On n'héberge pas quelqu' un que l'on soupçonne de vouloir vous voler ou vous assassiner.

Pourtant, c'est contraire aux lois anciennes et surtout à l'esprit de l'hospitalité.

Il semble que dans l'Antiquité on considérait que celui qui bénéficiait de l'hospitalité s'engageait à ne pas nuire à son hôte. Le temps de l'hospitalité êtait un temps de paix.

Cette notion ne paraissait pas aller de soi au XVIIIème siècle puisqu'on demandait au voyageur de prouver qu'il n'avait pas de mauvaises intentions.

Enfin, la troisième règle fait référence à la nécessité. Kant basait la nécessité sur le danger couru par le voyageur, les encyclopédistes semblent la baser sur la pénurie.

On est seulement tenu de recevoir s'il n'y a nul autre abri, et on peut même se dégager de cette obligation si, en payant, on trouve un autre logement pour le voyageur. Si l'on s"en tient à cette règle et surtout si on avait quelque argent, les cas dans lesquels il y avait obligation de recevoir etaient bien rares.

Paradoxalement, l'analyse de ces règles nous renvoie à la première définition soit. que l'hospitalité est une vertu, puisque, si on les applique, on peut presque toujours se dispenser de la domer. Donc, la donner relève bien de la volonté et en fait un acte vertueux.

Les auteurs indiquent aussi que, si l'hospitalitế naturelle s'est perdue, c'est parce que stoute l'Europe est devenue voyageante et commerçantex, c'est-à-dire que déjă le progrès des transports avait incité beaucoup de gens à voyager et fait naître de toutes parts des facilités d'hébergement qui ne rendaient plus le recours à l'hospitalité privéc aussi indispensable. C'était également que, devant le nombre de voyageurs qui auraient sollicité 1 'hospitalitế, la bonne volonté reculait.

D'autres grands textes nous décrivent avec beaucoup de précisions ce que fut $l^{\text {*accueil }}$ dans l'histoire des hommes.

\section{Un regard biblique}

Le texte appelé «LaPhiloxénied'Abraham» est particulièrement explicite. L'Éternel sous la forme de trois anges annonce à Abraham que malgré le grand âge de sa femme Sara, il sera Père.

... Quand il les vit, il courut audevant d'eux, depuis l'entrée de sa tente, et se prosterna en Terre. Et il dit: Seigneur... Permettez qu'on apporte un peu d'eau pour vous laver les pieds; et reposez-vous sous cet arbre. J'irai prendre un morceau de pain pour fortifier votre coeur... Abraham alla promptement dans sa tente vers Sara, et il dit: vite, trois mesures de fleur de farine, pétris et faitdes gâteaux. Et Abraham courut à son troupeau, prit un veau tendreet bon, et le donna à un serviteur qui se hẳta de l'apprểter. Il prit encore de la crème et du lait, avec le veau qu'on avaitapprêté, et ill le mit devant eux... (Gen 18 2-8)

Tout l'accueil est dans ces lignes.

Abraham reconnaît les anges, ils sont les envoyés de l'Éternel. Pour bien montrer qu'il a reconnu en eux l'Éternel, il se prosteme. Puis, il les traite selon les loisantiques de l'hospitalité. Il les fait se reposer sous l'arbre et non seulement leur donne un morceau de pain pour les réconforter mais encore fit préparer un festin: des gâteaux et un veau, grand sacrifice car c'est se priver de la virtualité d'avoir, plus tard, un boeuf, grand sacrifice en cette période difficile. Après la reconnaissance, l'hospitalité et lé maternage, il leur lave les pieds. Nous retrouverons dans toute l'antiquité ce rite du lavement des pieds qui a un usage pratique: on voyageaità pied et, à l'issue d'une longue marche, un bain de pieds étaitce que l'on pouvait offrir de mieux pour délasser celui qui arrivait. Et il a aussi une signification symbolique: on se courbe devant celui que l'on reçoit pour lui montrer qu'on l'aime et qu'on est prêt à l'aider. Saint Augustin a écrit: lorsqu'un chrétien s'incline vers les pieds de son frère, son coeur se penche aussi vers lui.

Ce n'est qu'après que les voyageurs aient été récconfortés, reposés, nourris, qu'on en vient à l'objet de leur visite: avant tout, il fallait accueillir dignement.

Un peu plus loin, nous voyons Lot assis à la porte de Sodome. Selon la coutume, il attend les voyageurs à la porte de la ville pour être sûr d'être le premier à leur pro- 
poser l'hospitalité et pour être sûr qu'aucun voyageur ne restera sans abri. Deux anges arrivent... Quand Lot les vit, il se leva pour aller au-devant d'eux et se prosterna la face contre Terre. Puis, il dit: «Voici mes Seigneurs, entrez je vous prie, dans la maison de votre serviteur, et passez-y la nuit; lavez-vous les pieds; vous vous lèverez de bon matin et vous poursuivrez votre route. Non, répondirent-ilis, nous passerons la nuit dans la rue. Mais Lot les pressa tellement qu'ils vinrent chez lui et entrèrent dans sa maison. Il leur donna un festin et fit cuire des pains sans levain. Et ils mangèrent... (Gen 19 2-6)

Et plus tard, alors que la foule de Sodome veut s'emparer des voyageurs (envoyés de Dieu), Lot les défends, propose mềme ses filles pour calmer les agresseurs et dit: *... ne faites rien à ces hommes puisqu'ils sont venus à l'ombre de mon toit...s (Gen 1911 11)

Les trois concepts de l'accueil se retrouvent aussi dans ce texte.

Lot se prosterne en signe de reconnaissance. Les rites de l'hospitalité et du maternage sont respectếs: le lavement des pieds, le gîte et le festin que l'on prépare spécialement. Il est à noter que les anges semblent vouloir décliner l'invitation de Lot, que peut-êttre ils jugeaient de pure forme et s'apprêtaient à passer la nuit dehors comme tous les voyageurs qui ne recevaient pas l'hospitalité. Lot insiste pour bien montrer sa volonté d'accueillir ceux qu'il a reconnu comme les envoyés de Dieu.

Enfin, il va jusqu'à proposer de sacrifier ce qu'il a de plus cher: ses filles pour calmer la foule et protéger les anges et cela non pas parce qu'ils sont anges mais parce qu'ils résident chez lui. C'est le devoir de protection dont se sent investi tout hôte vis-à-vis de ses invités.

\section{L'Odyssée}

Ce récit contient de nombreuses situations d'accueil où nous retrouvons nos grands principes.

Au chant premier, Télémaque se plaint que l'on ait fait attendre Pallas à la porte du Palais - "Après l'avoir salué et invité à se restaurers, il mena la déesse s'asseoir dans un beau fauteuil bien incrustê sur lequel il avait êtendu une housse de lin et sous ses pieds il mit un escabeau... Une servante apportant dans une belle aiguière d'or de l'eau pour les mains, la leur versait audessus d'une cuvette d'argent et disposait devant eux une table polie. Une digne intendante leur apporta le pain et leur servit en abondance des mets qu'elle avait en réserve. L'écuyer tranchant leur tendit des plateaux de viandes diverses, posa devant eux des coupes d'or et maintes fois l'échanson venait leur verser le vin (I 122.135).

On voit que l'on sortait pour le visiteur ce qu'il y a de plus beau, la vaisselle d'or et d'argent, la housse de lin, l'escabeau sous les pieds; les mets qu'on avait en réserve et ce qu'il y a de meilleur: on le nourrit avec les viandes et le vin. Auparavant, Télémaque lui-même avait veillé au confort de l'invitée. Son accueil est un tel événement que toute la maisonnée en est bouleversée.

Plus tard, quand Pallas Athénée et Télémaque arrivent à Pylos chez Nestor, celuici, après les avoir accueilli à sa table et restauré s'enquéra du motif de leur visite. "Il serait plus honnête, maintenant que nos hôtes se sont réjouis à manger, de les questionner, de nous informer de leur nom: étrangers, qui êtes-vous? Est-ce une affaire qui vous amène ou bien errez-vous sur la mer, sans but, en pirates qui voguent à l'aventure et apportant le malheur?\% (III 70)

Ce passage indique que l'hospitalité était accordée à tout voyageur quel qu'il soit. Le voyageur doit être reçu et, les rites accomplis, on voit après qui il est et si ses motifs de voyage sont honnêtes. Dans tous les cas on satisfait aux obligations.

Par ailleurs, quand Athénée et Télémaque décident de coucher à bord de leur nef, Nestor les supplie de n'en rien faire, «Zeus et tous les autres Dieux immortels m'épargnent cet affront que vous vous en alliez de chez moi dans votre vaisseau... Tant que moi je vivrais et qu'après moi resteront dans mon logis des fils pour héberger les hôtes qui viendront ici...* (III 345.358)

Il y a là concordance avec le récit biblique. Nestor comme Lot ne peuvent souffrir qu'on refuse leur hospitalité. Cela les empêcherait d'être vertueux. Par ailleurs, cela aurait pu signifier que leur maison n'était pas digne de recevoir les voyageurs de passage. Donc pour lui comme pour Nestor, se voir refuser l'hospitalité constitue un affront.

Par ailleurs, la notion de respect de la personne humaine est particulièrement mise en avant quand Ulysse, sous l'apparence d'un pauvre hère, arrive chez les Porcher Eumée et y est très gentiment reçu et dit: *Que Zeus et les autres Dieux immortels t'accordent, mon hôte, ce que tu désires le plus, car tu m'as accueilli dans ton coeurs.
Et celui-ci répond: «Étranger, je n'ai pas le droit, quand mểme viendrait quelqu'un de plus mésireux que toi, de manquer de respect envers un hôte. Ils sont tous envoyés par Zeus, étrangers et mendiants...\% (XIV $5457)$

Il y a dans ces mots toute la mystique de l'accueil. L'un est réconforté parce que, malgré son apparence, on l'a aimé, etl'autre répond: tu aurais pu être encore plus laid, je t'aurais reçu et aimé tout pareil car en toi comme en tous les voyageurs, je reconnais celui qui est protégé par Dieu: l'Homme. Et c'est au nom de cette mystique toujours vivante qu'aujourd'hui encore, personne ne peut supporterd'être mal accueilli. Nous voulons que derrière notre apparence ou noscomportements, celui qui accueille voit l'homme.

\section{On retrouve aussi dans 1'Odyssée:}

- la notion de protection du voyageur, ou,ce qui revient au même, la réprobation du mal, fait à l'invité - quand Ulysse rentre chez lui déguisé en mendiant et qu'il est agressé par Antinoüs, tous les autres prétendants blâment ce dernier avec violence: antinoüs, ce n'est pas beau: tu as frappé un pauvre errant, imprudent!» (XVII 4...) Le mot «imprudents montre bien que nuire à un hôte de passage est assimilé à un sacrilège. Quand Pénélope apprend qu'on avait frappé un mendiant chez elle, elle déclare: "Qu'ainsi te frappe toi-même Appolon à l'arc fameux» (XVII 482). Celui qui se conduit mal avec l'étranger fûtil fils de Dieu, est maudit. Héraclès, auteur des grands travaux, qui a tué Iphitos alors qu'il était son hôte, est traité d'insensé, car il n'avait pas craint la «colère vengeresse des dieux, sans respect de cette table où ill'avait reçu» (Iphitos) (XXI 18/19).

- et le rite du lavement des pieds ou, mieux encore, le Bain - quand Télémaque reçoit Athénée, il la prie de rester en disant: "Quand tu auras pris le bain et fait bonne chère ici, tu regagneras ton vaisseau» $(1-300)$. C'est la belle Polycaste, fille de Nestor, qui reçoit Télémaque et «lorsqu'elle l'eut baigné et frotté d'huile fluide, elle jeta sur lui une tunique...* (III 465). Ulysse, racontant l'accueil de Circe, précise: \&... Quand l'eau eut bouilli dans le bronze luisant, elle me fit entrer dans la baignoire, et après avoir doucement attiédi l'eau du grand trépied, elle m'en lavait la tềte et les épaules pour chasser de mon corps la fatigue qui ronge le coeur. Et puis, m'ayant lavế et frotté d'huile fluide, 
elle me revêtit d'un beau manteau pardessus une tunique.s $(X$ 351.360)

Homere nous apprend que les cadeaux d'hospitalité tenaient une grande place dans les rites d'accueil. On donnait au voyageur selon ses besoins et son rang social, de la nourriture, des vêtements, des armes ou des objets précieux.

Télémaque dit à Athénée: «Quand... tu regagneras ton vaisseau, la joie au coeur, avec un présent magnifique, précieux, tel que les hôtes en donnent à des hôtes aimes; tu le garderas en souvenir de moi\% (I 300.304). Ménêlas promet à Télémaque atrois chevaux et un char bien poliw et ajoute: «je te donnerai encore une belle coupe, afin qu'il te souvienne de moi quand chaque jour tu feras des libérations aux Dieux immortels... (IV 575.577)

Enfin, quand chez les Phéaciens, Ulysse est reconnu comme homme de qualité, Alcinoùs déclare: adonnons-lui un présent d'hospitalité comme il sied. Il y a dans le pays douze Rois fameux qui agissent en souverain, et je suis moi le treizième. Apportez chacun un manteau bien lavé, une tunique et un talent d'or précieux ${ }_{n+\ldots}$ (V) (VIII 384.392)

Les cadeaux d'hospitalité semblent avoir eu deux fonctions principales:

- honorer celui que l'on reçoit et le remercier d'avoir permis à l'hôte d'accomplir une action conforme à la morale;

- crécr le souvenir, et éventuellement, lui rappeler qu'il a une dette d'hospitalité.

On retrouve encore très exceptionnellement l'esprit antique des cadeaux d'hospitalite dans les voyages de Chefs d'Etat où celui qui reçoit remet à son visi= teur des cadeaux pour lui signifier qu'il a été honoré par sa visite.

Si les traditions de l'hospitalité des temps héroïques semblent s'être quelque peu perdues en Grèce à partir du IVème siècle avant J -C. et à Rome où l'hospitalité commerciale des auberges et des hôtelleries (de bien mauvaise qualité, tous les historiens en conviennent) s'est rapidement imposce, elles s'etaient, en revanche, maintenues dans d'autres pays. César dit dans ses commentaires que les gemains regardaient comme un crime de faire quelque outrage aux étrangers. Il ajoute que quand il en venait chez eux, pour quelque cause que ce fut, ils empêchaient qu'on les insultât, et les regardaient comme des personnes sacrées, toutes les maisons leur étaient ouvertes et partout on leur donnaità manger. En Gaulle, non seulement on hébergeait le voyageur, mais on le conduisait et on veillait à sa sécurité. Si sur la grande voie herculéenne qui allait d'Italie en Espagne un voyageur souffrait quelque injure ou dommage, on punissait tous ceux sur le territoire desquels la chose était arrivée.

On dit même que chez les Celtes, le meurtre d'un étranger était plus sévèrement puni que celui d'un citoyen.

\section{Les pèlerinages}

Jusqu'au Xlème siècle, chez certains peuples d'Europe Centrale, on considérait que refuser l'hospitalité à un ếtranger était un crime capital.

C'est toutefois dans le monde chrétien des IVème et Vème siècles que l'accueil s'organise.

Lespèlerinages incitaient de plus en plus de fidèles à voyager et à visiter les lieux saints. Le Clergé d'Orient eut donc I'obligation morale de veiller sur ceux qui étaient sur les routes.

Les pèlerins ne sont pas loin de considérer qu'ils ont droit à l'hospitalité des religieux, suivant en cela l'enseignement des apôtres qui faisaient de l'hospitalité un devoir sacré.

Les évêques vont prendre une part importante à l'organisation matérielle de l'hébergement des voyageurs. Basile de Césarée fut le premier à créer des hôtelleries pour les pèlerins, les axenodochias, que l'on appellera pendant longtemps, en souvenir de leur créateur, les «Basiliades\%.

Le concile de Nicée en 325 substitue la loi à l'initiative privée en exigeant qu'il y ait dans chaque ville des wxenodochias et des «Hospitias (autre forme d'hôtellerie religieuse).

Malgré l'existence de ces institutions, les évêques durent continuer à prêcher les vertus hospitalières.

Le mêrite consisteàs s'occuper soi-même de l'étranger, et Jean Chrysostome dit qu'un bon chrétien doit faire de sa maison un «xenodochium\%.

Mais ce sont surtout les moines qui sont à cette époque les spécialistes de l'hospitalité. Les règles de Pakhôme, de Basile, et les lettres de Jérôme montrent tous les cas que font de l'accueil les grands chefs du monachisme.

Tous les monastères ont un kxenodochi- ums ou un pavillon des hôtes, ce qui n'est pas sans poser de problème. Les voyageurs empêchent souvent les moines de trier et de travailler, et, parailleurs, les frais d'entretien de ces établissements sont quelquefois si élevês, car l'hospitalité y était entièrement gratuite, que les religieux doivent faire des prodiges et même s'endetter pour les faire vivre.

Les formes de l'accueil y sont traditionnelles à une exception près. II est indispensable que le nouveau venu prouve sa bonne foi catholique. Pakhôme est formel; il n'accepte que ceux qui professent la même foi que lui et ses cénobites. Cette peur de 1 'hérétique est un trait caractéristique de la chrétienté du IVème siècle, comme on ne se fie guère à une déclaration orale, on exige un témoignage écrit. Ainsi, s'institue l'usage d'une sorte de passeport, lettre de recommandation, qui joue le rôle des atesseres d'hospitalitéx.

La littérature ecclésiastique signale l'existence de lettres de communion - slitterae communi catoriacs ou lettres formées «litterae formatae* sans doute réservées à lahiérarchie de l'Église etde simples letures ecclésiastiques: epistolae pour les laïques. Ces lettres, une fois produites et la question d'orthodoxie réglée, le voyageur est reconnı comme un frère et l'hospitalité reprend ses droits.

Si les moines sont prévenus de l'arrivée d'un personnage important, ils se portent à sa rencontre et vont 1 'accueillir dès qu'on le voit. En signe d'humilité, on lui lave les pieds et on va parfois comme aux temps héroïques jusqu'à les lui oindre d'huile parfumée. Ensuite on apporte des rafraichissements, du pain, des fruits et du vin aromatisé.

Mais les moines rendent encore d'autres services aux visiteurs. Pour ceux qui voyagent sans escorte et sans domestiques, l'Église remplit tous les rôles. Dès l'instant où ils sont admis dans le pavillon des hôtes, ils n'ont à se soucier de rien, on leur fournit de l'argent s'ils en ont besoin, on renouvelle leurs habits et on les nourrit de la meilleure façon. Les règlements monastiques prévoient un moment pendant lequel al'hôteliers doit venir converser avec les hôtes et Jean Chrysostome ecrit: «Il ne faut pas que celui qui est reçu ait l'impression qu"il est de trop dans la maison, ou mểne qu'on le néglige si peu que ce soibs.

En cas de maladie, le voyageur est soigné, veut-il faire des excursions, les moines servent de guides, on s'occupe aussi de la suite de son voyage. On met à sa disposition chevaux et conducteur et on se charge aussi de lui réserver un logement pour sa 
prochaine étape: c c'est un véritable service de luxe.

Mais le souci de subvenir aux nécessités matérielles de leurs hôtes n est pas le seul, pour les moines. Ils cherchent surtout à atteindre leurs âmes. L'étranger, dès qu'il a franchi le seuil de l'hôtellerie, est invité à se tourner vers Dieu et à prier.

Le haut Moyen-Age et le Moyen-Age furent des époques vouées au respect et à I'assistance du voyageur, une ère d'hospitalité et de secours. Par endroits, l'accueil était, comme on dit aujourd'hui, l'affaire de tous. Chez les Bourguignons, par exemple, celui qui recevait un étranger était dédommagé par ses concitoyens, chacun pour sa quote-part et celui qui refusait l'hospitalité était mis ả l'amende. Il est vrai qu'à cette époque, tavernes et hồtellerie sont devenues des repaires de brigands et des lieux de débauche. Les récits qui en font foi sont innombrables, et de très peu nombreux règlements durent être pris pour sauvegarder ce qui restait de la morale. Le concile de 847 statua sur les peines encourues par les religieux qui fréquenteraient ces établissements. Une ordonnance de 1315 précise que sl'hoste qui retient les effets d'un étranger mort chez lui, doit rendre le triple de ce qu'il a retenus. Une autre du 27 février 1350 dit formellement que les taverniers «ne doivent recevoir ni receler chez eux aucun joueur de dés et autres gens diffamés... et ne peuvent recevoir des buveurs chez eux lorsque le couvre-feu de Notre-Dame sera sonné...n.

Pendant toute cette longue période, qui va de la fin de l'empire Romain à la Renaissance, on a surtout recours à l'hospitalité privée, et les autorités religieuses et temporelles ne cessent d'inciter les populations à recevoir et à aider les voyageurs.

Charlemagne, dans ses capitulaires, renouvelle sans cesse les instructions relatives aux hospices et à l'hospitalité: l'article 16 de celui de 803 dit que l'hospitalité ne doit jamais être refusée au voyageur, l'article 35 de celui de 809 précise que I'on doit asile à tous les voyageurs.

Théodule écrit: all est recommandé aux prêtres d'aimer l'hospitalité et de ne la refuser à personne... Il y a de l'inhumanité et de la barbarie à ne pas accueillir un etranger....

Certains textes tentent de remettre en honneur les usages anciens: *Que l'hospitalité antique ne soit pas mise en oubli, qu'on ne néglige pas de recueillir des Pẻlerins et de leur laver les pieds, qu'on reçoive miséricordieusement tousceux qui se présenteront suivant la règle et la coutume de nos prédécesseurs... \$ (supplique conservée au chapitre XXXII du livre III des Antiquités de Fulde par Christian Domer).

Le succès des grands pèlerinages comme celui de Saint Jacques de Compostelle, dont les fameux chemins sont établis au XIIème siècle, est pour beaucoup dans l'extension du souci de l'accueil des voyageurs.

\section{Le voyageur est traité comme un ami}

Dans des textes du XVeme siècle, on trouve aussi de fort bonnes descriptions de réceptions réservếes aux êtrangers.

Erasme, dans un de ses colloques, par la voix de Guillaume décrit avec force détails l'accueil dans une auberge.

Personne ne saurait être mieux traité chez soi qu'à Lyon dans une auberge. Premièrement, 1 'hôtesse, qui a l'air de quelque chose, venait nous voir, nous disant de nous réjouir et nous priant de prendre, en bonne part, ce qu'on mettait sur la table. À la mère succédait la fille, fort jolie personne (...) Ce qui faisait le plus de plaisir, c'est que ces demoiselles ne nous parlaient pas comme à des étrangers, comme à des passagers qui logent chez elle, mais elles s'entretenaient aussi librement avec nous que si nous avions été le plus ancienset les plus intimes amis de la maison (...) Comme ces aimables personnes ne pouvaient pas être toujours avec nous, ayant leurs occupations domestiques, etd'ailleurs étant obligées de visiter aussi et de saluer les autres passagers, on nous laissait, pour nous tenir compagnie et nous divertir, une jeune fille instruite etaguerrie (...) je puis vous assurer qu'on mange très bien et si bien que je conçois pas comment ils traitent de cette abondance et de cette forme là pour un prix si médiocre: on croirait qu'ils y mettraient du leur, et qu'ils viseraient plutồt à pratiquer la vertu d'hospitalité qu'à vivre commodément de leur négoce (...) Pour moi, je m'imagine être au logis plutôt qu'en voyage ou en auberge (...) Ces prétendues pucelles vous demandaient librement și vous n'aviez rien de sale, et si on leur donnait des hardes à laver, elles les rapportaient dans toute la propreté souhaitable...

Ce petit texte met en avant les valeurs éternelles de l'accueil. Le voyageur' est traité comme un ami, un membre de la famille. On veille à son confort moral en le distrayant et à son confort physique en le nourrissant bien. L'auteur introduit d'ailleursà ce sujet non seulement la notion d'honnêteté mais encore presque une notion de sacrifice. L'hôte semble en donner plus que pour l'argent qu'il reçoit. Le abon accueil\% est réussi car le visiteur se sent aussi bien que chez lui.

Montaigne semble lui très sensible au confort matériel et à la bonne chère. Dans son * journal de voyage en Italie par la Suisse et l'Allemagnew, on peut lire: «à dire vrai, j'ai toujoursété non seulement bien, mais même agréablement logé dans tous les lieux où je me suis arrêté en Italiè. A Mulhouse, son arrivée ayant été signalé au seigneur du lieu, on wva vers lui - et - la seigneurie fit ses honneurs à MM d'Estissac et de Montaigne que de leur envoyer par l'un de leurs officiers, de leur vin avec une longue harangue....

En Toscane, il retrouve la coutume monastique de prier le passant d'accepter l'hospitalité: «ils ont ceste coustume ecrit-il = d'envoyer au-devant des étrangers sept ou huit lieues les éconjurer de prendre leur logis. Vous trouverez souvent l'hoste même à chevals.

Mabillon, un bénédictin, nous donne aussi dans le récit d'un voyage qu'il fit dans les Alpes en 1682, quelques détails sur l'accueil dans les auberges: alorsqu'on arrive dans une auberge, 1 'hôte et l'hôtesse vous tendent la main, et vous assurent qu'il ne pourrait venir personne chez eux qui leur fut plus agréable.... et un peu plus loin, il signale que: aau moment du départ, c'est une coutume de porter aux partants une santé pour l'amour de Saint-Jean».

A Pariségalement, on trouvait d'excellentes auberges. L'hôtesse la plus célèbre de la fin du XVIle était sans doute La Boisselière. Établie près du Louvre, toute la haute noblesse défilait chez elle. Elle avait la réputation d'être la plus joyeuse hồtesse de Paris et xil valait mieux - disait-on - faire station chez elle, qu'antichambre chez le Roiss. Le Fermier Gếnếral Monsieur de Béchamel, Marquis de Niontel, venait y déclamer wex-professos des leçons de Haute Gastronomie et y inventa la célèbre sauce.

\section{Un accueil particulier}

L'histoire nous livrequelques anecdotes où sont mentionnées des façons d'accueillir qui, si elles nous paraissent acurieusess dans leurs formes, n'en releve pas moins des concepts fondamentaux de $1^{\prime}$ accueil.

Jean-François Regnard dans son «voyage 
en Laponies raconte que parti pour Torno le 23 juillet 1681, il s'arrêta dans une Maison où il fut l'objet selon l'habitude d'un accueil particuliêrement chaleureux.

Je vous ai remarqué, Monsieur, que les étrangers ont en ce pays un grand privilège qui est d'honorer les filles de leur approche. Ils en ont un autre qui $n^{\dagger}$ est pas moins considérable, qui est de partager avec les lapons leurs lits et leurs femmes. Quand un étranger vient dans leurs cabanes, ils le recoivent le mieux qu'ils peuvent, et pensent le régaler parfaitement s'ils ont un verre d'eau de vie à lui donner. Mais après le repas, quand la personne qu'ils reçoivent est de considération, et qu'ils veulent lui faire chère entière, ils font venir leurs femmes et leurs filles, et tiennent à grand honneur que vous agissiez avec elles comme ils le feraient eux-mêmes; pour les femmes et les filles, elles ne font aucune difficulté de vous accorder stout ce que vous pouvez souhaiters, et croient que vous leur faites autant d'honneur qu'à leurs maris ou à leurs pères.

Le récit se poursuit par l'aventure d'un français qui, quelques années auparavant, parce qu'il avait refusế les faveurs de la femme, fut enfermé avoc celle-ci, par le mari jusqu'à ce qu'il lui fasse l'amour, et par celle d'un certain Joannes Tornoeux, prêtre des lapons qui, devant les suppliques d'un mari qui pensait que cela lui attirerait. les bienfaits de Dieu, dut se résoudre à coucher avec sa femme afin - dit-il - de ne pas le désespérer.

Le récit est corroboré par Kaj Birket-Smith qui fut Directeur du Musée National de Copenhague et qui rapporte également que chez les Aléoutes, peuple esquimau, «Les bonnes manieres exigent... que les hommes mettent leurs femmes à la disposition de leurs hôtes... $\%$.

C'est peut-être au Moyen-Orient que les traditions de l'hospitalité antique se sont maintenues le plus longtemps. Un touriste anglais, $\mathrm{Mr}$ Buckingham, raconte comment, dans les années 1850, en Égypte, il passa plusieurs jours chez un hôte qui insista pour l'hếberger.

Nos chameaux furent déchargés au Khan, mais les nombreux amis de notre compagnon de voyage ne nous permirent pas de rester là. Ils nous invitèrent avec tant d'insistance que nous crûmes d'abord nécessaire de refuser a tous pour prévenir toute jalousie. Pendant une journée entière, nous recûmes les visites des habitants les plus respectables de la ville, et le soir... Nous nous rendìmes dans une maison où l'on avait préparé a souper. Nous fûmes recus dans une chambre magnifique ornée de lambris dores, de sièges couverts, de riches tapis avec des coussins de soie, Deux fakirs, mendiants indiens étaient assis avec lesautres sur le sofa, et quoique vêtus de haillons et couverts de vermine, ilsétaient servis avec la meme attention que nous...

Nous retrouvons dans ces lignes les grands principes de l'accueil Homérique. On est honoré de recevoir un étranger et on met tout en oeuvre pour que son séjour soit agréable: bonne chère, visites, etc. Mais aussi, l'accueil n'est pas réservé aux personnes de qualité; on reçoit des mendiants et on les traite avec autant d'égards que les grands personnages.

A traversces quelquesexemples, nous avons pu voir que, sous des formes différentes, on retrouvait toujours dans l accueil au cours des temps nos trois concepts fondamentaux: reconinaissance, hospitalité, maternage. Ils sont, évidemment, encore présents dans l'accueil moderne.

\section{Le grand tournant}

Mais le grand basculement s'est produit vers le milieu du XIXeme siècle avec la naissance de l'accueil professionnel. Ce basculement a été dû à:

1) la presque totale disparition de l'hospitalité prives:

2) la mise en place d"une hottellerie de qualité qui a rendu l'hospitalité commerciale ${ }_{+}$shonorables.

Les hôteliers et les professionnels de l'accueil ont repris à leur compte les lois de l'hospitalitế et les vertus de l'accueil. Ils se sont fait les gardiens de la tradition antique et ont fait leur le code qui régissait l'hospitalité privée. Il y a eu transfert des obligations: les particuliers se sont déchargés sur eux de toutes leurs obligations d'accueil.

On a vu apparaittre vers 1850 de grands pionniers de l'hôtellerie comme Ober, Seiler, les frères Knechtenhofer, Johan Strübin, Maximilien de Pfyffer d'Altis-Hofen, Joseph Durrer, Michel Zufferey et celui qui deviendra le plus célèbre: César Ritz.

Ils se sont tous, eux et leurs successeurs, sentis investi d'une responsabilité et d'une mission humaine: celle de veiller sur leurs frères en déplacement.

Ils ont mis au point des techniques d'accueil w, de façon à ce que les hommes, dont les attentes n'ont pas changé depuis la nuit des temps, soient aussi à l'aise dans leurs établissements que ce qu'ils étaient auparavant dans des maisons amies.

Ils ont posé les bases de cette fantastique évolution qui permet aujourd' hui à des centaines de milliers de professionnels de recevoir chaque année des centaines de millions de voyageurs.

L'accueil dans 1'hôtellerie de luxe est directement issu de l'accueil privé traditionnel.

Les Palaces sont les derniers temples des grandes traditions de l'accueil et c'est souvent d'eux que l'on s'inspire quand on veut créer des lieux d'accueil de grande qualité. C'est d'ailleurs dans les hồtels que la nécessité d'un renouveau de l'accueil s'est d'abord fait sentir et c'est dans l'hottellerie ques'est amorcé ce quel'on pourrait appeler une renaissance de l'accueil traditionnel.

Le savoir-faire que nous appelons le philoxénologie deviendra peut-être un jour une science. C'est en parti grâce à luí que les hommes ont pu vivre en société et que les civilisations ont prosperét. Notre société moderne, malade de sa croissance, ne survivra que si elle arrive à développer dans 1'homme le sens de l'accueil comme nos anciens ont su le faire. $f$

\section{Notes explicatives}

(1) La cite antique Pars 1864

(2) Projet de pao perpetuelle.

(3) L'encyclopedie, sorte de dictionnaire qui avait pour but de reunir l'ensemble des connaissances de l'epoque a été conçue par les libraire Le Breton, et retalise de 1751 a 1772 en 35 volumes; sous la direction de Diderot entouré de 150 collaborateurs dont Montesquieu, Rousseau, Voltaire. d'Alembert, Helvetris, Condillac etc. 